The ASTROPHYSICAL JouRnal, 261:L117-L120, 1982 October 15

(1) 1982. The American Astronomical Society. All rights reserved. Printed in U.S.A.

\title{
THE ABUNDANCE OF THE ACTINIDES IN THE COSMIC RADIATION AS MEASURED ON HEAO 3
}

\author{
W. R. Binns, ${ }^{1,2}$ R. K. Fickle,${ }^{3}$ T. L. Garrard,${ }^{4}$ M. H. Israel,${ }^{1}$ J. Klarmann, ${ }^{1}$ E. C. Stone, ${ }^{4}$ and \\ C. J. WADDINGTON ${ }^{3}$ \\ Received 1982 January 29; accepted 1982 June 30
}

\begin{abstract}
The $H E A O 3$ detector of heavy cosmic-ray nuclei has observed one possible actinide nucleus and some 100 nuclei of the platinum-lead group of elements. The resulting upper limit of $3 \%$ for the abundance ratio of actinides to platinum-lead nuclides is significantly lower than previous results from other observations. This new limit is inconsistent with freshly synthesized, pure $r$-process sources for cosmic-ray nuclei in this charge interval but is consistent with a source having a composition similar to the solar system, or to aged $r$-process material. We observe no events with a charge greater than 96 .
\end{abstract}

Subject headings: cosmic rays: abundances - nucleosynthesis

\section{INTRODUCTION}

The actinides, which can only be synthesized by the rapid addition of neutrons to lighter seed nuclei (the $r$-process or $n$-process), include the long-lived elements $\mathrm{Th}$ and $\mathrm{U}$ as well as the shorter lived species $\mathrm{Np}, \mathrm{Pu}$, and $\mathrm{Cm}$ which may also survive for the cosmic-ray lifetime of $\sim 10^{7} \mathrm{yr}$ if freshly synthesized just prior to acceleration. The report by Fowler et al. (1967) that the actinide abundances were significantly greater in the contemporary cosmic radiation than in solar system matter suggested that the source of cosmic-ray nuclei was enriched in the products of $r$-process nucleosynthesis. Such a suggestion was consistent with cosmic rays originating in supernovae or supernova remnants where $r$-process enrichment might be expected and where the energetics of cosmic-ray acceleration could be plausibly explained.

Later observations with balloon-borne detectors (Fowler et al. 1977) and on the Skylab mission (Shirk and Price 1978) appeared to confirm this suggestion. A typical value of $10 \%-20 \%$ for the ratio of "actinides," defined here as nuclei with $88 \leq Z \leq 100$, to "platinum-lead," $74 \leq Z \leq 87$, was generally accepted and was consistent with some of the predictions for $r$-process material. However, Meyer (1979) did point out that these data lacked internal consistency and could also be interpreted as implying a much lower ratio.

In this Letter we report results from the detector of heavy nuclei flown on the $H E A O 3$ spacecraft. Although

\footnotetext{
${ }^{1}$ Department of Physics and the McDonnell Center for the Space Sciences, Washington University, St. Louis, Missouri.

${ }^{2}$ Formerly at McDonnell Douglas Research Laboratories, St. Louis, Missouri.

${ }^{3}$ School of Physics and Astronomy, University of Minnesota, Minneapolis, Minnesota.

${ }^{4}$ California Institute of Technology, Pasadena, California.
}

the analysis of all the data is not complete and we have not yet calibrated our instrument with beams of energetic particles having charges comparable to those of the ultraheavy cosmic-ray nuclei $(Z \geq 30)$, we have exhaustively combed the data for potential "actinide" events. As a result, we have established an upper limit to the abundance of the actinides that is significantly lower than abundances reported earlier, thus altering the conclusions drawn from the earlier observations on the nature and origin of the cosmic-ray source material.

\section{THIS OBSERVATION}

The instrument flown on the $H E A O 3$ spacecraft (Binns et al. 1981b) consisted of ionization chambers, multi-wire ionization hodoscopes, and a Cerenkov detector. This instrument was designed to respond to energetic nuclei having charges, $Z$, between about 14 and 120 and to have sufficient collecting power to observe $\sim 5 \times 10^{4}$ iron nuclei per day with $E \gtrsim 350$ $\mathrm{MeV}$ per amu. The minimum energy that a nucleus of $Z=78$ required in order to give a measurable Cerenkov signal, a prerequisite for a multiparameter charge determination, was about $500 \mathrm{MeV}$ per amu, corresponding to a rigidity of $\sim 2.7 \mathrm{GV}$. This threshold, combined with the orbit characteristics, means that at least $80 \%$ of the observed nuclei with $Z \geq 70$ have energies $>1 \mathrm{GeV}$ per amu.

HEAO 3 was launched on 1979 September 20 into a $500 \mathrm{~km}, 43.6$ orbit and operated until 1981 May 30 . We report here results from the first 450 days of operation. We have previously published results on nuclei with $Z \leq 40$ (Binns et al. 1981a), based on which we showed that, for a selected subset of the data, we resolve the individual even-charged nuclei and that the charge scale did not deviate significantly from a strict $Z^{2}$ dependence 


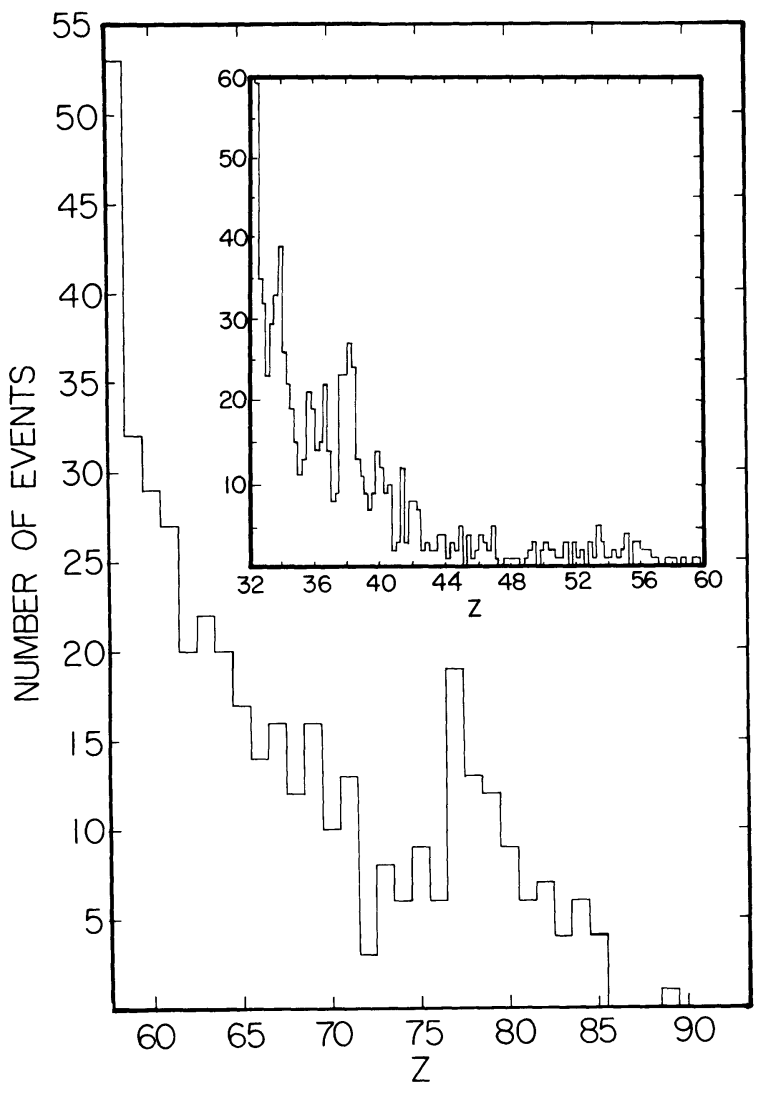

Fig. 1.-Observed number of events vs. assigned charge, $Z$. The main part of the figure shows all events from 450 days of data for which a charge assignment can be made; the charge resolution of this set does not permit separation of abundances of individual elements. The inset shows a selected subset for which individual element abundances can be determined for even- $Z$ elements at least to $Z=42$.

on either the Cerenkov or ion chamber signals for $Z \leq 40$. The inset in Figure 1 shows data similar to that of Binns et al. (1981a) but drawn from a longer interval.

In order to maximize the number of very high charge nuclei $(Z \gtrsim 70)$ available for detailed analysis, it was necessary to relax the selection criteria used at lower charges. The consequent degradation in charge resolution is compensated by the increased sample size. In measuring the abundance ratio of the "actinides" to the "platinum-lead" nuclides, we are aided by the relative absence of the short-lived radioactive nuclides in the actinide "gap" $(84 \leq Z \leq 89)$. This allows us to accept a considerable degradation in charge resolution of the "platinum-lead" nuclei without there being an appreciable spillover into the "actinides."

The triggering requirements for our instrument consisted of demanding significant signals from any two of the seven charge-measuring detectors (six ion chambers and the Cerenkov counter) and any two hodoscopes. For each recorded event, the particle trajectory was determined from the hodoscope, and geomagnetic cut-off rigidities were estimated for the two possible directions of motion at the particular orientation of the instrument in space. Some of the events were then rejected as unsuitable for further consideration. For example, events occurring during passage through the South Atlantic Anomaly were rejected due to the high background rates from trapped protons. Events having low cut-off rigidities $(<4 \mathrm{GV})$ were rejected if they lacked either a Cerenkov or an ion signal because estimates of $Z$ are very uncertain for low-energy particles without both signals. Events were also rejected if there was no minimal consistency between the separate Cerenkov signals or the contiguous ion chambers. However, these latter rejected events were assigned charge estimates, and direct examination of those with apparent $Z \geq 58$ showed that none are true actinides. Finally, events were rejected if accidental triggering of widely separated hodoscope elements led to an ambiguous particle trajectory.

Figure 1 shows 1 "actinide" and 101 nuclei with $74 \leq Z \leq 87$ leading to a significantly lower value for the "actinide" to "platinum-lead" ratio (henceforth the actinide ratio) than previously published. To prevent a selection bias against actinides, the data in Figure 1 came from a reanalysis by hand of every event that was assigned a nominal charge of $Z \geq 58$ for any reason by the various computer algorithms. The events rejected by this detailed hand analysis were found to be spillover from the more abundant lower charges whose high charge had been erroneously estimated by simple computer algorithms. These rejection criteria show no significant charge dependence over the entire range.

Figure 1 shows charges based on estimates that are extrapolated from iron assuming that both the ion chamber and Cerenkov signals scale as $Z^{2}$. For these high- $Z$ nuclei, higher order $Z$ terms would be expected to become increasingly important as the assumption of the first Born approximation that $Z / \beta \ll 137$ breaks down. A summary of the theory of these higher order terms has been given by Ahlen (1980), while Derrickson, Eby, and Watts (1981) have calculated the effects that might be observed in our particular detectors. These higher order corrections, if applied in our data analysis, would have the effect of lowering our estimated charges and so would only decrease the number of actinides. However, the validity of these corrections applied to a specific detector is not fully understood, and ultimately a calibration using known particles will be essential to a complete analysis of the observations. For data with a broad energy distribution, the assumed $Z^{2}$ scaling may have shifted and smeared the charge spectrum to some degree. The concentration of events in the "platinumlead" region, the sharp drop in abundances just beyond $Z \approx 38$ and again beyond $Z \approx 58$ shown in Figure 1 , are expected from both $r$-processes and $s$-processes. This indicates that our charge assignments are not grossly in 
error. The continuous distribution to $Z=85$ was expected as a consequence of the effects of the relativistic $1 !$ rise in ionization of lower charge nuclei, just as the distribution in the vicinity of $Z=60$ is expected from relativistic rise from the peak around $Z=56$.

Our data directly indicate an actinide ratio, of $\leq 0.01$, with an upper limit of 0.03 corresponding to an $84 \%$ confidence level if the one particle is an actinide. We have not observed any events with $Z>96$ and therefore place an upper limit on the "super-heavy" to "platinum-lead" abundance ratio of 0.03 with $95 \%$ confidence.

\section{DISCUSSION}

Figure 2 compares this $H E A O$ value of the actinide ratio with other reported observations. Our result is inconsistent with the results from balloon-borne plastic-track and nuclear emulsion detectors (Fowler et al. 1977) and with those of the Skylab plastic-track detector (Shirk and Price 1978). Our value is consistent with the results of the Ariel 6 experiment (Fowler et al. 1981), although we set a much more stringent upper limit on this ratio.

The progressive reduction in the reported values of the actinide ratio is what would be anticipated from a progressive improvement in the experimental resolution of the detectors. Because of the extreme rarity of the actinides, it is essential that lighter nuclei not be misidentified as actinides because of a fluctuation in response or a nuclear interaction. The multiparameter analysis in our experiment, in which several measurements are made in each of two different kinds of detector (ionization and Cerenkov), provides internal consistency checks on individual events which reduce accidental misidentifications. Thus the HEAO detector would be expected to give better separation of actinide and lead nuclei than any of the other detectors, and the lower value found for the actinide ratio can be understood as a reduction in the degree of spillover from a relatively abundant platinum-lead region.

Our future analysis of the $H E A O$ data cannot be expected to show an appreciable change in the actinide ratio. Detailed analysis of the lower resolution data sets may result in a slight increase in the number of "platinum-lead" nuclei, but it will not produce any more actinide nuclei, since for this time period all potential candidates have already been analyzed. The additional data from the remaining days can improve our statistics by only $\sim 25 \%$. Clearly, any future experiment to measure this ratio should be designed to observe at least an order of magnitude more particles with a charge resolution that is as good as or better than that of the $H E A O$ detector.

The observed actinide ratio is directly related to the nature of the $r$-process contributions to cosmic-ray

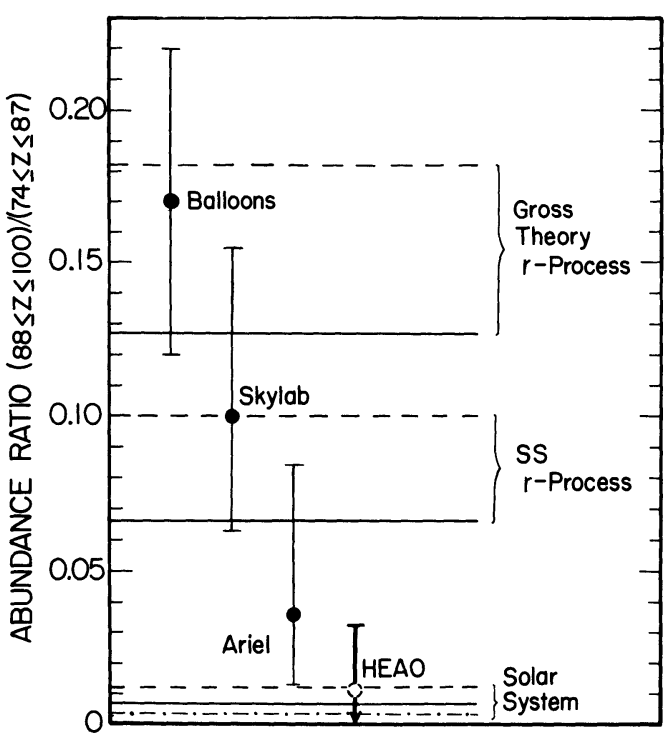

FIG. 2.-The abundance of "actinides" relative to "platinumlead" as reported by various observers and as predicted under several assumptions. Data points: Balloons (Fowler et al. 1977), Skylab (Shirk and Price 1978), Ariel (Fowler et al. 1981), HEAO (this paper). Theoretical lines (solid and dashed): (Blake et al. 1978). The effects of corrections due to first ionization potential are shown as the dashed lines in each case. Dot-dashed line (Brewster, Freier, and Waddington 1981, 1983) is for a source with composition of solar system today, rather than solar system at the time of its formation.

source material. This ratio is only weakly dependent on assumed interaction cross sections both in the detector and in the interstellar medium, due to the narrow mass range and the small relative abundances of the actinides. The actinide ratio is thus a good test of the validity of the initial assumptions regarding the source. Various source distributions have been assumed for the actinide ratio with the resulting propagated values shown in Figure 2.

Attempts to fit the earlier observations were based on the assumption of a cosmic-ray source composed of freshly synthesized $r$-process material. In Figure 2 the curve labeled "SS $r$-process" assumes a cosmic-ray source with abundance ratios corresponding to the production ratios that would have led (in a specific model of galactic chemical evolution) to the $r$-process abundances subsequently observed in the condensed bodies of the solar system (Blake et al. 1978). These "SS $r$-process" abundances are similar to those derived by Blake et al. from an $r$-process theory based on the Senbetu $\beta$-decay theory. A theoretical $r$-process based on the Gross theory of $\beta$-decay gave Blake $e t$ al. an even higher actinide ratio. Our result is the first that is clearly inconsistent with these predictions of a freshly synthesized $r$-process source. 
Our actinide result is consistent with solar system source abundances. However, it cannot distinguish between solar system and aged $r$-process abundances. If instead of freshly synthesized $r$-process, the cosmic-ray source looked like the $r$-process part of the solar system (i.e., non-freshly synthesized material), the actinide ratio would be less than a factor of 2 higher than the solar system ratio, well within our measurement limit.
We thank T. J. Aufrance, B. W. Gauld, D. P. Grossman, J. Hanlon, M. D. Jones, K. E. Krombel, and D. P. Mitchell for assistance in programming for data analysis and R. A. Mewaldt for useful discussions. The research was supported in part by NASA under contracts NAS 8-27976, 77, 78 and grants NGR 05-002-160, 24-005-050, and 26-008-001.

\section{REFERENCES}

Ahlen, S. P. 1980, Rev. Mod. Phys., 52, 121.

Binns, W. R., Fickle, R. K. Garrard, T. L., Israel, M. H., Klarmann, J., Stone, E. C., and Waddington, C. J. $1981 a$, Ap. J. (Letters), 247, L115.

Binns, W. R., Israel, M. H., Klarmann, J., Scarlett, W. R., Stone, E. C., and Waddington, C. J. 1981 b, Nucl. Instr. Meth., 185, 415.

Blake, J. B., Hainebach, K. L., Schramm, D. N., and Anglin, J. D. 1978, Ap. J., 221, 694.

Brewster, N. R., Freier, P. S., and Waddington, C. J. 1981, Proc. 17th Int. Cosmic Ray Conf. (Paris), 2, 40, and private communication.

Cation. 1983, Ap. J., 264, in press.
Derrickson, J. H., Eby, P. B., and Watts, J. W. 1981, Proc. 17th Int. Cosmic Ray Conf. (Paris), $8,88$.

Fowler, P. H., Adams, R. A., Cowen, V. G., and Kidd, J. M. 1967, Proc. Roy. Soc. London, A, 301, 39.

Fowler, P. H., Alexandre, C., Clapham, V. M., Henshaw, D. L., O'Sullivan, D., and Thompson, A., 1977, Proc. 15th Int. Cosmic Ray Conf. (Plovdiv), 11, 165.

Fowler, P. H., Walker, R. N., Masheder, M. R., Moses, R. T., and Morley, A. 1981, Nature, 291, 45.

Meyer, J. P. 1979, Proc. 16th Int. Cosmic Ray Conf. (Kyoto), 1, 374.

Shirk, E. K., and Price, P. B. 1978, Ap. J., 220, 719.

W. R. Binns, M. H. IsRael, and J. KLARmann: Department of Physics, Washington University, St. Louis, MO 63130

R. K. Fickle and C. J. Waddington: School of Physics and Astronomy, University of Minnesota, 116 Church Street, S. E., Minneapolis, MN 55455

T. L. GarRaRD and E. C. Stone: 220-47 Downs Laboratory, California Institute of Technology, Pasadena, CA 91125 